1

2

3

\title{
The use of atypical antipsychotics and the risk of breast cancer
}

Laurent Azoulay $\mathrm{PhD}^{1,2}$, Hui Yin $\mathrm{MSc}^{1}$, Christel Renoux MD $\mathrm{PhD}^{1}$, Samy Suissa $\mathrm{PhD}^{1,3}$

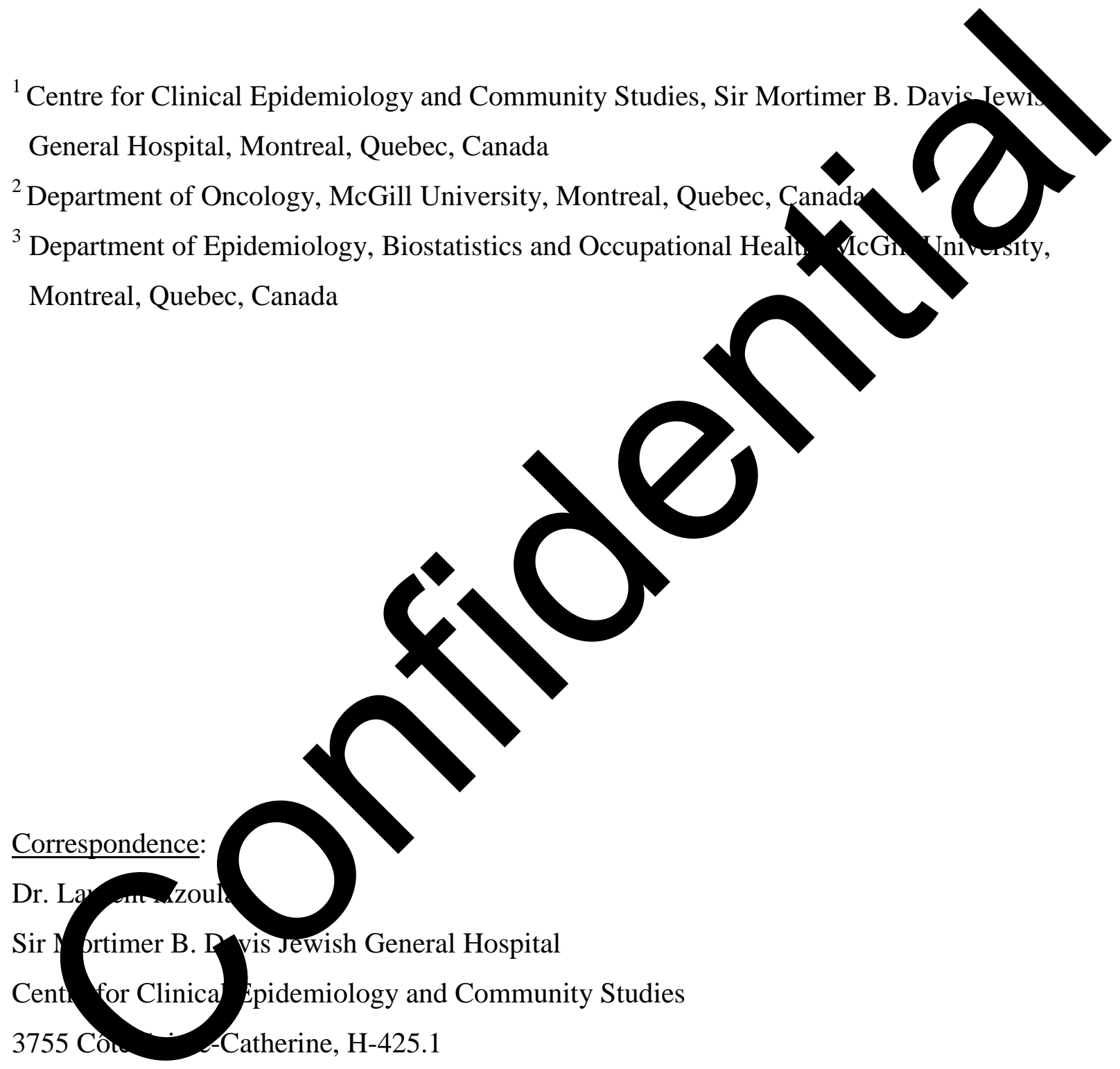

Montreal, Quebec, Canada, H3T 1E2

Tel: 514.340.8222 extension 8396

Fax: 514.340.7564

Email: laurent.azoulay@mcgill.ca 


\begin{abstract}
Purpose: To determine whether atypical antipsychotics, when compared to typical antipsychotics, increase the risk of breast cancer.

Methods: We conducted a retrospective cohort study using a nested case-control analysis within the United Kingdom General Practice Research Database population. We identified a female patients prescribed at least one antipsychotic (either typical or atypical), betwee an 988 and December 31, 2007, with follow-up until December 31, 2010. All incidentca s of rast cancer were identified and matched up to 10 controls. Adjusted rate os (R) breast cancer associated with ever use of atypical antipsychotics was compar tover us typical antipsychotics.

Results: The cohort included 106,362 patientesc presc rate: 2.7 per 1000/year). Compare to patien who on pused typical antipsychotics, exclusive users of atypical antipsychotics we not an creased risk of breast cancer (RR: $0.81,95 \%$ CI: $0.63,1.05)$. These results re ant ans considering specific atypical antipsychotics known to significantly incro prolacm levels such as risperidone (RR: 0.86, 95\% CI: 0.60, 1.25). Furthermor no dos esponse was observed in terms of cumulative duration of use and cumy rive dose in o ne equivalents.

Con sion: The fults of this study should provide reassurance that compared to typical antipsychon, atypical antipsychotics do not increase the risk of breast cancer.
\end{abstract}

Keywords: Antipsychotics; Breast cancer; Population-based 


\section{Introduction}

Antipsychotics are now playing important role in the treatment of several psychiatric disorders. In fact, there has been a significant increase in their use, particularly for off-label indications [1,2]. Despite their effectiveness, antipsychotics frequently cause side effects, including hyperprolactinemia [3-5]. High serum prolactin levels are associated with instrual irregularities, galactorrhea, gynecomastia, sexual dysfunction, infertility and de mineral density [4]. In addition, some evidence suggests that antipsychotigs, vur th rr ef ws on elevating prolactin levels, may increase the risk of breast cancer [6] is poto ial risk was known for first-generation (typical) antipsychotics, as these ha show increase prolactin levels in a dose-dependent fashion [7]. While second neratior (atypical) antipsychotics have been associated with lessextrap ami prolactin elevations [8], there have been renewed conce not be necessarily the case, especially for some of the newer sical an sychot such as risperdone and amisulpride which have been associated with oh pro lence of severe hyperprolactinemia $[4,7,9]$. To date, few observa the astia ave investigated the assotion between antipsychotics and the incid e of broast cancer. While most of these studies found null effects [10-14], they had number methodological limitations. First, many of these studies were
antip chotics in $t$ market. Second, some of these studies were not able to distinguish between
the effects treatment from that of the underlying disease [10,11,13,15]. Patients with chronic psychiatric disorders are followed more closely than the general population, and it is thus possible that any increased risk is partly due to surveillance bias. In one study, a modest association was observed for antipsychotics in relation to breast cancer risk (HR: 1.16, 95\% CI: 
$1.07,1.26)[15]$. However, antipsychotic users were compared to non-users (mainly non-diseased individuals), raising the possibility that confounding by indication or surveillance bias may affected the results. Furthermore, that study did not differentiate between the use of typical versus atypical antipsychotics, and no analysis was undertaken to assess individual antipsychotics, such as risperidone and amisulpride, as these agents significantly ele prolactin levels $[4,7,9]$.

Given the increasing use of the newer atypical antipsychotics, and lack of a o meir long-term safety, more research is needed to determine whether thes rents cease the risk of breast cancer. Thus, the objective of this large population-base otu was etermine whether atypical antipsychotics, in comparison to typical antipsychotic ncrease ne risk of breast cancer.

\section{Methods}

Data source This study was cond General Practice Research Database (GPRD), a primary care database from Uniteamingdom (UK) [16]. The GPRD is the world's largest computerized date ase of gitudinal records from primary care. It contains the complete the 4 population enrolled in more than 600 general practices. The geographic distribution of population, and age and sex distributions of patients in the GPRD are similar to those reported by the National Population Census [17]. Participating general practitioners have been trained to record medical information including demographic data, medical diagnoses, and procedures 
using a standardized form. Prescriptions dispensed by GPRD physicians are automatically transcribed into the computer record. In addition, the GPRD collects information regarding lifestyle variables such as body mass index (BMI), and quantitative and qualitative data pertaining to smoking and excessive alcohol use. The Read classification is used to enter medical diagnoses and procedures, and a coded drug dictionary based on the UK Prescription icing Authority Dictionary is used for recording prescriptions. The recorded informat protocol was approved by the Independent Scientific Advisory Comp oe on GrR and the Ethics Committee of the Jewish General Hospital.

\section{Study population \& study design}

We conducted a population-based cob within the GPRD population. The onot co isted of female patients who received at least one prescription for any antipsych (eith typical or atypical), between January 1, 1988 and December 31, 2007, with for unt vecember 31, 2010.

Cohort entry was tho of a Mrst prescription for an antipsychotic (either typical or atypical) during th study p iod.Patients were required to have at least one year of up-to-

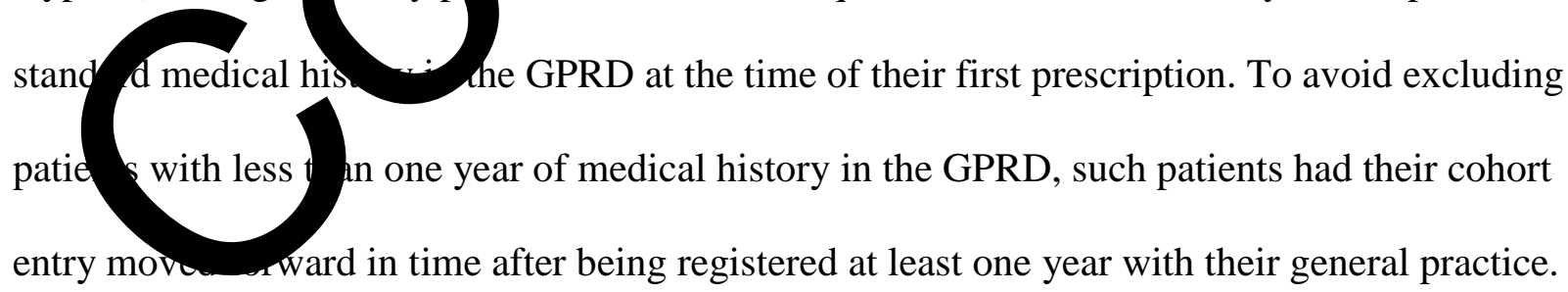
This cohort entry definition led to the inclusion of both incident and prevalent antipsychotic users. These two groups were differentiated by determining whether there was exposure to antipsychotics in the year prior to cohort entry. 
The cohort was restricted to patients at least 18 years of age at the time of cohort entry. Patients with a history of breast cancer at any time prior to cohort entry were excluded (identified using the algorithm described below). The latter criterion was necessary to identify incident cases of breast cancer during follow-up. Thus, all patients in the cohort were followed until a first-ever diagnosis of breast cancer, death from any cause, end of registration with th reneral practice, or end of the study period (December 31, 2010), whichever came first

\section{Case-control selection}

From the cohort of patients described above, we identif ata incida ases of breast cancer using a validated computerized algorithm created with he context a previous study on hormone replacement therapy and the risk of breast PEGASUS and Read codes for breast cancer 1 as medical procedures, visits, or treatments related to thi urcome. hese co sist of mastectomies, lumpectomies, axillary node dissections, consulta ss wit ncologists, chemotherapy treatments, radiotherapy and use of postoperative ant one ther $95 \%$ of breast cancer diagnoses identified with this algorithm were col rmed in previous review of written records of a random sample of 100 cases [22]. (he cale ar arte of each case's event was defined as the index date. up to 10 con randomly selected from the case's risk set, after matching on year of bi year of co prt entry, prevalent use of antipsychotics, and duration of follow-up. To avoid excrum o cases, we relaxed the matching criteria for 8 cases to year of birth \pm 1 year and year of cohort entry \pm 1 year. By definition, all controls were alive, never diagnosed with breast cancer, and were registered with their general practice when matched to a given case, and thus 
had equal duration of medical history information at the risk set date. The date of the risk set was the index date for the controls.

\section{Exposure to antipsychotics}

We considered all antipsychotics on the UK market during the study period. h typical antipsychotics that were considered consisted of benperidol, chlorpromazine, flupentixol, fluphenazine, fluspirilene, haloperidol, levomepromazine, loxapin pericyazine, perphenazine, pimozide, pipotiazine, promazine, sulpirt thiort vine, trifluoperazine, trifluperidol, and zuclopenthixol. Prochlorperar ate, wicas not included since it is used as a treatment for migraines, nauso and moning sickness in the UK. However, patients who used prochlorperzine to ther antipsychotic at cohort entry were included in the cohort. The atypicen syat were considered consisted of amisulpride, aripiprazole, clozapistanza ve, quet sine, remoxipride, risperidone, sertindole, and zotepine.

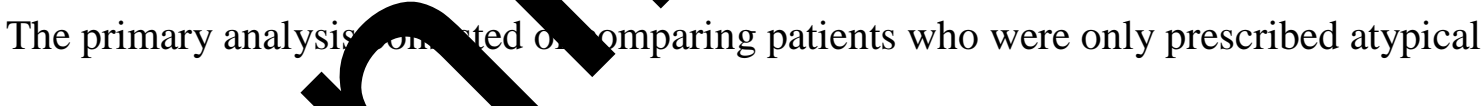
antipsychotics to patients on prescribed typical antipsychotics between cohort entry and index

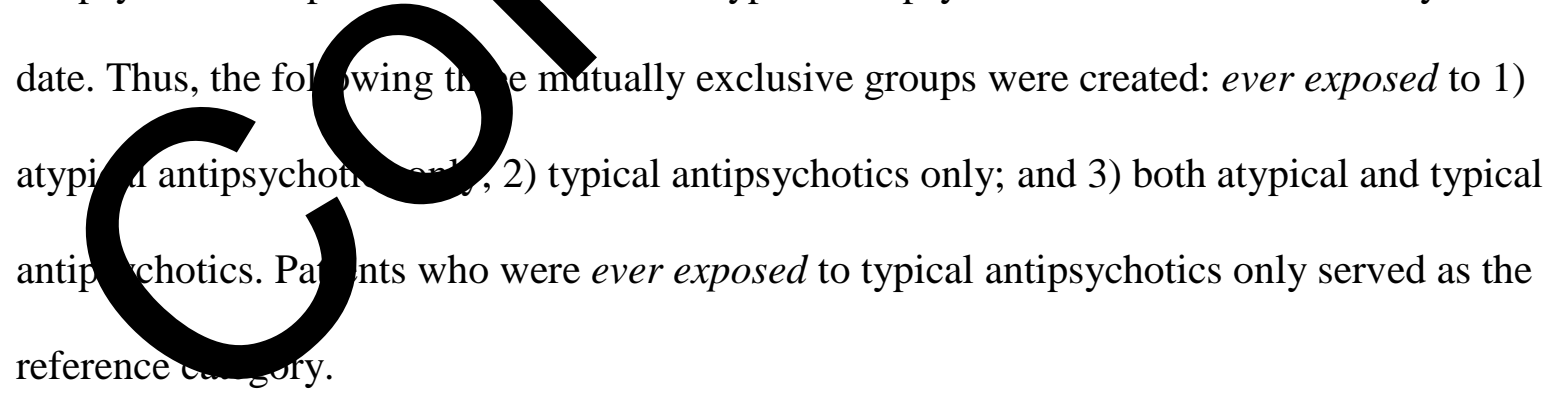

In a subsequent analysis, we determined whether specific atypical antipsychotics, particularly risperidone (the most frequently prescribed atypical antipsychotic in our population), increased the risk of breast cancer. Therefore, patients ever exposed to atypical antipsychotics 
risperidone only, 2) risperidone and other atypical antipsychotics, and 3) other atypical antipsychotics.

Finally, we conducted two dose-response analyses among patients ever exposed to atypical antipsychotics only: cumulative duration of use and cumulative dose. Cumut ve duration of use was calculated by summing the durations of all atypical antipsy prescriptions up until the index date for each patient. As for cumulative dose, we ff st co merted all atypical antipsychotic prescriptions to olanzapine milligram equir nts [2. These equivalents were then summed for each patient up until the ind ration of use and cumulative dose were entered in tertiles in the moda based on the distribution in the controls. For all exposure definitions above, ye excl for a biologically meaningful latency time wir

\section{Antipsychotics with prolactin o}

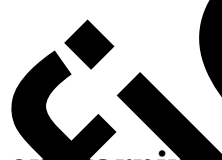

There are a number anch known to increase prolactin levels, as indicated on their warning labels. These 'sist of amisulpiride, benperidol, chlorpromazine, fluphenazine, haloperidol, olanz fine, pe henazine, pericyazine, pimozide, pipotiazine, risperidone, sulpiride, triflu erazine and z thixol. Therefore, it was of interest to determine whether such antip chotics inc ased the risk of breast cancer, compared to antipsychotics with no such warning lave patients were categorized into one of the following three mutually exclusive groups: ever use of 1) antipsychotics with warning labels only, 2) both antipsychotics with and without warning labels, and 3) antipsychotics with no warning labels only. The latter group served as the reference category for this analysis. 


\section{Potential confounders}

The risk estimates were adjusted for co-morbid clinical conditions and exposures, measured at index date, known to be associated with breast cancer that might also influence the choice of antipsychotic therapy. These consisted of excessive alcohol use, obesity (BMI $\geq 30$ ), smoking status, aspirin use, selective serotonin reuptake inhibitors, statins, previous (other than non-melanoma skin cancer and breast cancer), hypertension, insulin other oral hypoglycaemic agents, prior oophorectomy, prior use of hormone repla men werapy (HRT), and prior use of oral contraceptives. Finally, in order to mint a any tentual effect of confounding by indication, we adjusted the models for known chot atcations. These consisted of schizophrenia and related disorders, bipolar disoro other psychotic disorders, dementia, major depression with or without peychoti feat end others.

\section{Statistical analysis}

Descriptive statistics were d to marize the characteristics of the cohort, cases and

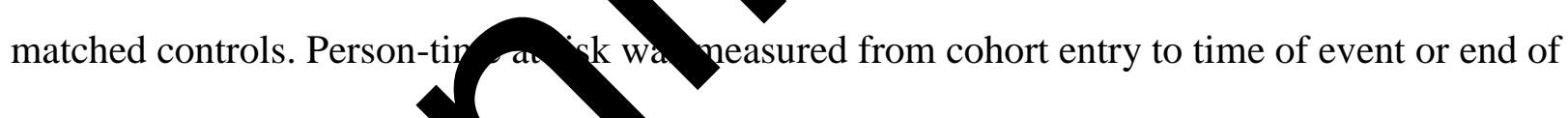
follow-up. Conditional logi regressron was used to estimate RRs, along with 95\% confidence intervals (CI). In dition the be birth, year of cohort entry, prevalent antipsychotic use and
potential cy founders described above. atypical antipsychotics only when compared to ever use of typical antipsychotics only. Since our cohort also included prevalent users, we conducted a sensitivity analyses by stratifying cases and matched controls on the prevalent use of antipsychotics prior to cohort entry. 
We conducted three secondary analyses among patients ever exposed to atypical antipsychotics, one of which determined whether the risk of breast cancer was increased in patients exposed to risperidone, and two others to evaluate whether the risk increased in a dosedependent fashion according to cumulative duration of use and cumulative dose.

We also conducted two exploratory analyses to determine whether breast can risk varied between different patient groups. In the first analysis, we assessed wheth menopausal status modified the association between atypical antipsychotigs and b ast a percer. This analysis was performed because several epidemiologic studies between serum prolactin levels and breast cancer risk in pre- ar pond women [2427]. Thus, we stratified cases and matched controls based on a at cohor entry $(\geq 50$ versus < 50) as a proxy for menopausal status. In the sacond a alys stratified cases and matched controls based on their history of HRT use, on therapy has been shown to increase prolactin levels [24].

Finally, we conducted ano anals to determine whether patients prescribed

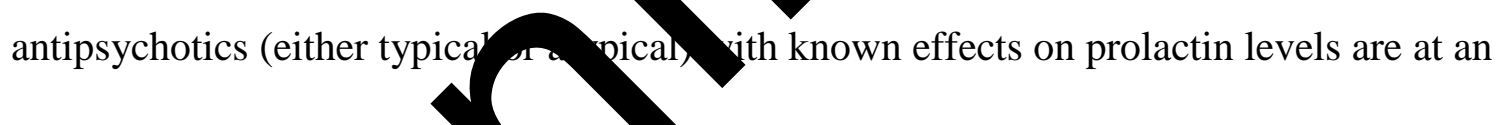
increased risk compared to ients wh. did not use such drugs. All analyses were conducted

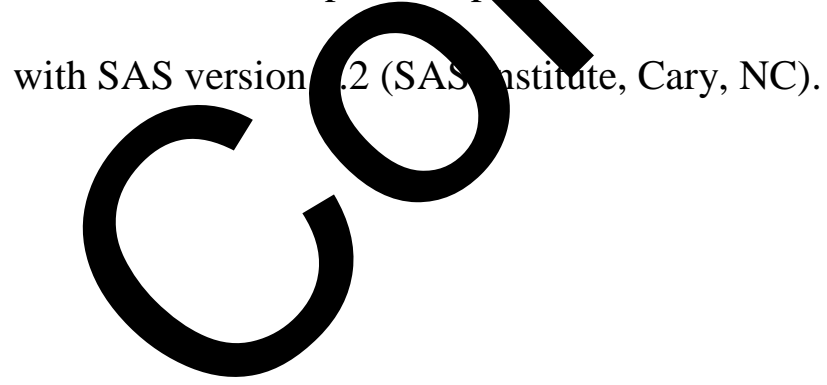




\section{Results}

Of the 139,863 female patients using antipsychotics during the study period, 106,362 met the inclusion criteria (Figure 1). The mean (SD) age at cohort entry was 63 (21.6) years, and the mean duration of follow-up was 5.3 (4.8) years. At cohort entry, 85,142 (80.0\%) were prescribed typical antipsychotics, 20,800 (19.6\%) were prescribed atypical antipsychotics, whild $\$ 0(0.4 \%)$ were using both concomitantly. Of patients prescribed typical antipsychotics at thioridazine (36.4\%) was the most frequently prescribed, while risperidone was th mos frequently prescribed $(47.3 \%)$ atypical antipsychotic.

At the time of cohort entry, 20,241 (19.0\%) patients we an nosed in major depression with or without psychotic features, $9646(9.1 \%)$ wh dementid, $7472(7.0 \%)$ with psychotic disorders, $5683(5.3 \%)$ with schizonhrenia 
categorized by drug type, no increased risk was found among those prescribed risperidone (Table 2). With respect to cumulative duration of use and cumulative dosage of atypical antipsychotics, there were no statistically significant associations, although the point estimates were lower than one in the former (Table 3).

The results of the secondary analyses indicated that breast cancer risk did not fer significantly between pre- and post-menopausal patients. Similarly, past use of a no appear to modify the risk, although the adjusted RR for HRT users was hioher na for n pr-users (adjusted RR: 0.99, 95\% CI: 0.56, 1.75 and adjusted RR: 0.76, 95\% $0.57,03$, respectively) (Figure 2). Finally, patients exclusively prescribed antipsychot on to prolactin levels were not an increased risk of breast cancer, compared to ose who were not prescribed such drugs (adjusted RR: 1.06, 95\% CI: 0.921.22).
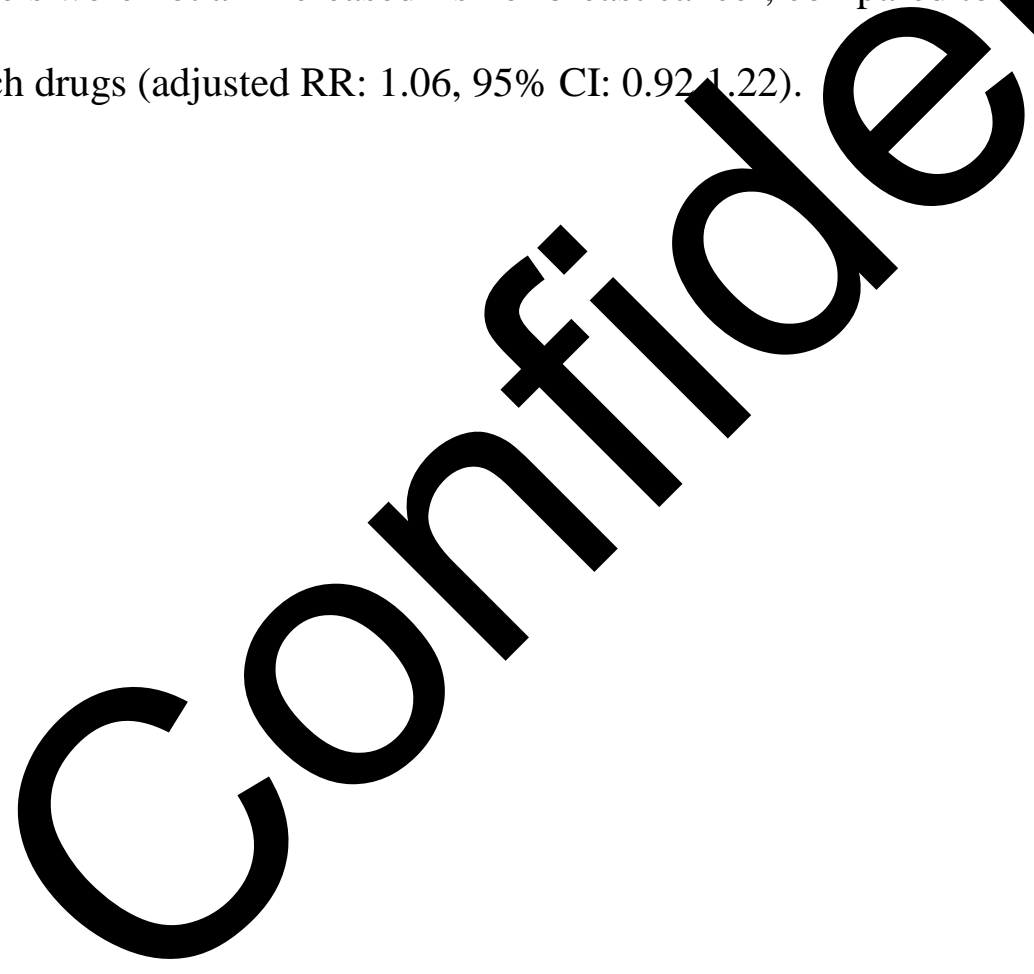


\section{Discussion}

The results of this study indicate that atypical antipsychotics do not increase the risk of breast cancer compared to typical antipsychotics. This finding was strengthened by the lack of any dose-response association, which considered both cumulative duration of use and cumulative dose. Furthermore, no increased risk was observed in high risk groups, such as in pos menopausal women and in those with a history of HRT use. Finally, no increas observed with antipsychotics known to increase prolactin levels, suggesting that se es vations do not translate into an increased breast cancer risk, compared to oth ntips, otics.

To our knowledge, this is the first study to investigate antipsychotic agents increase the risk of breast cancer. Our study provides re vuring e lidence that compared to typical antipsychotics, atypical antipsychorics do this in in patients exposed for up to 23 years. In fact, although not statisticall point estimates in the different analyses were all under unity, suresting th atypica mipsychotics, when compared to typical antipsychotics, might be associate ith a ver risk of breast cancer. Whether these effects are due to the anti-tumour prope certo atypical antipsychotics, or by a higher carcinogenicity of typical at sychoties remains to be determined. Thus, these results need to be Thus, the confirmed in large carefun designed studies. number of breast cancer cases. Second, because the GPRD uses pre-recorded exposure histories, the possibility of recall bias was eliminated. Third, our exposure and covariates were timedependent, thus taking into account changes in these variables over time. Finally, the GPRD 

database contains information on a number of important confounders, such as BMI, excessive alcohol use, and smoking. Therefore, we were able to adjust for a number of important confounders often absent in administrative databases.

This study does have some limitations. First, drug information in the GPRD represents prescriptions written by general practitioners. As such, it is unknown whether prescri actually filled at the pharmacy. Second, as with any observational study, confoy indication is always a concern. However, this potential bias was minimized by asi s a rence group consisting of antipsychotic users. Furthermore, we adjusted th odels the most common indications of antipsychotic use, to further reduce any est ql colding by indication. We were not able to adjust for certain breast cance $\mathrm{k}$ factors, such as family history of breast cancer, parity, and age at menarche variables were differentially distributed between atypic typsenotic users, lowering the possibility that these unmeasured arables sed the sults. Finally, it is possible that some physicians concerned with the pro in-elo ting potential of atypical antipsychotics preferentially prescribed typ at insyd to patients at high risk of breast cancer, which would have diluted the poin timate the null. Although this is a possibility, it is unlikely as atypical antipsych ics wen introduced in the market in the 1990s on the premise that they woul oe more effed ale producing less adverse effects than typical antipsychotics, altho this view as been challenged [28,29]. In mansion, the results of this study indicate that atypical antipsychotics, when compared to typical antipsychotics, do not appear to increase the risk of breast cancer. These results remained consistent after considering duration of use and dose, and different subgroups of 
patients at an inherently increased risk of breast cancer. These results should provide reassurance to both physicians and patients on the long-term safety of these agents.

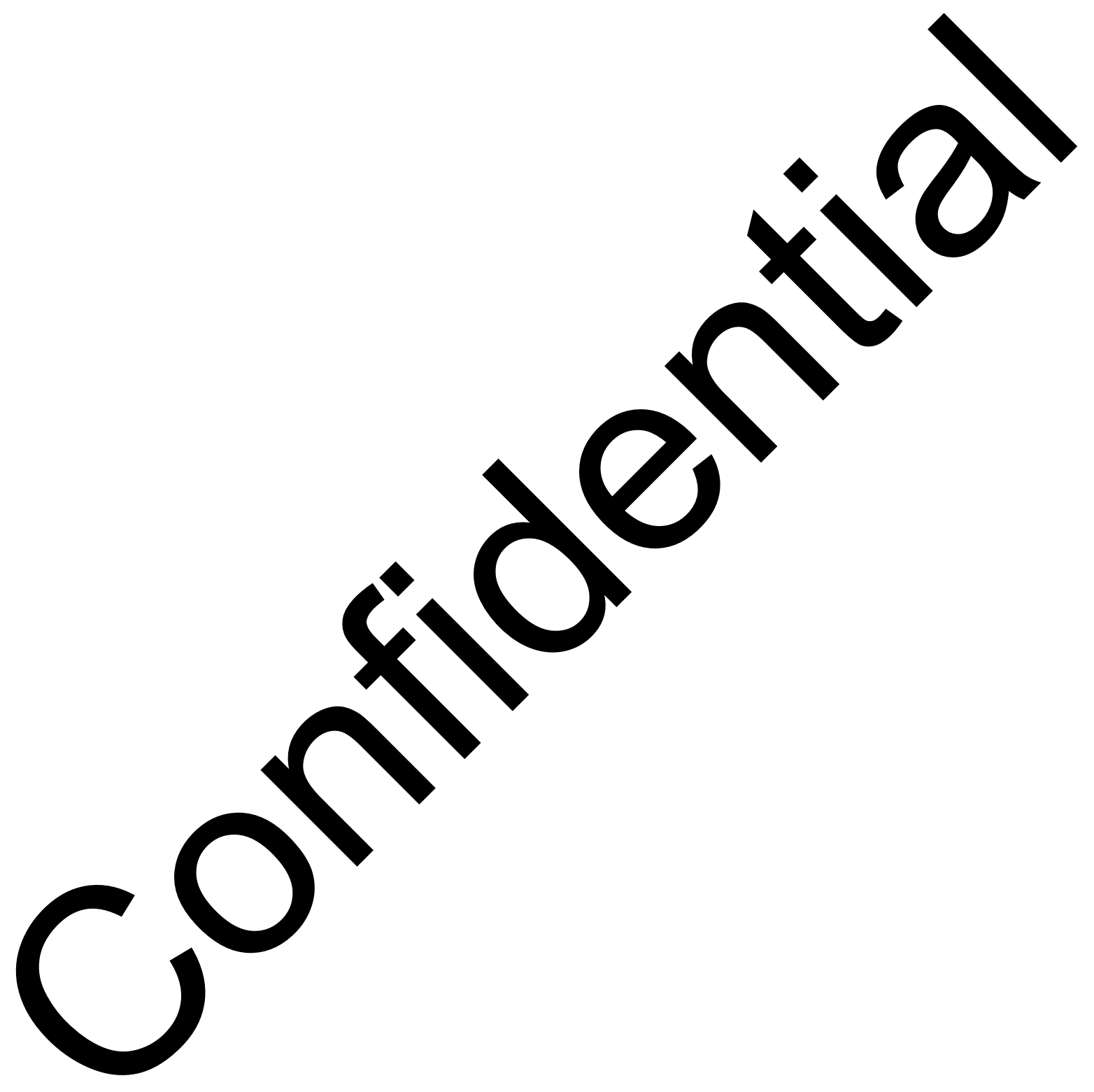




\section{Acknowledgments}

Dr. Laurent Azoulay is the recipient of a 'Chercheur-Boursier' Award from the Fonds de la recherche en santé du Québec. Dr. Samy Suissa is the recipient of a Distinguished Investigator Award from the Canadian Institutes of Health Research. This study was supported by a Catalyst Grant from the Canadian Institutes of Health Research.

\section{Conflicts of interest}

The authors report no conflicts of interest. 


\section{References}

1. Alessi-Severini S, Biscontri RG, Collins DM, Kozyrskyj A, Sareen J et al (2008)

Utilization and costs of antipsychotic agents: a Canadian population-based study, 19962006. Psychiatr Serv 59:547-553.

2. Leslie DL, Mohamed S, Rosenheck RA (2009) Off-label use of aripsyc ftic red tions in the department of Veterans Affairs health care system. Psyc Se 60: 1181.

3. Allison DB, Mentore JL, Heo M, Chandler LP, Cappe (JC et a Y999) Antipsychoticinduced weight gain: a comprehensive research nthy A. A Psychiatry 156:1686-1696.

4. Bostwick JR, Guthrie SK, Ellipgrod (2009) ntipsychotic-induced hyperprolactinemia. Pharmacotherapy 29:64-73

5. Miller CH, Mohr F, U brich Woerner M, Fleischhacker WW et al (1998) The prevalence of ramidal signs and symptoms in patients treated with clozapine, rispardone, d conve tional antipsychotics. J Clin Psychiatry 59:69-75.

6. ang PS, W ker AM, Tsuang MT, Orav EJ, Glynn RJ et al (2002) Dopamine antagonists and the development of breast cancer. Arch Gen Psychiatry 59:1147-1154.

7. Haddad PM, Wieck A (2004) Antipsychotic-induced hyperprolactinaemia: mechanisms, clinical features and management. Drugs 64:2291-2314. 
8. Seeman P (2002) Atypical antipsychotics: mechanism of action. Can J Psychiatry 47:27-38.

9. Bushe C, Shaw M, Peveler RC (2008) A review of the association between antipsychotic use and hyperprolactinaemia. J Psychopharmacol 22:46-55.

10. Overall JE (1978) Prior psychiatric treatment and the development of breastancer. ch Gen Psychiatry 35:898-899.

11. Wagner S, Mantel N (1978) Breast cancer at a psychiatric hacpital fore and after the introduction of neuroleptic agents. Cancer Res 38:270 08 . 
15. Wang PS, Walker AM, Tsuang MT, Orav EJ, Glynn RJ et al (2002) Dopamine antagonists and the development of breast cancer. Arch Gen Psychiatry 59:1147-1154.

16. Walley T, Mantgani A (1997) The UK General Practice Research Database. Lancet 350:1097-1099.

17. Garcia Rodriguez LA, Perez GS (1998) Use of the UK General Katice for pharmacoepidemiology. Br J Clin Pharmacol 45:419-425.

19. Lawrenson R, Williams T, Farmer R

18. Jick H, Jick SS, Derby LE (1991) Validation of inform on recond on general practitioner based computerised data resource in ary 302:766-768. general practice databases. Pubin - Yeak 1:299-304.

21.

20. Lawrenson R, Todd Leyo GM, LWilliams TJ, Farmer RD (2000) Validation of the diagnosis of ve thro boembolism in general practice database studies. Br J Clin

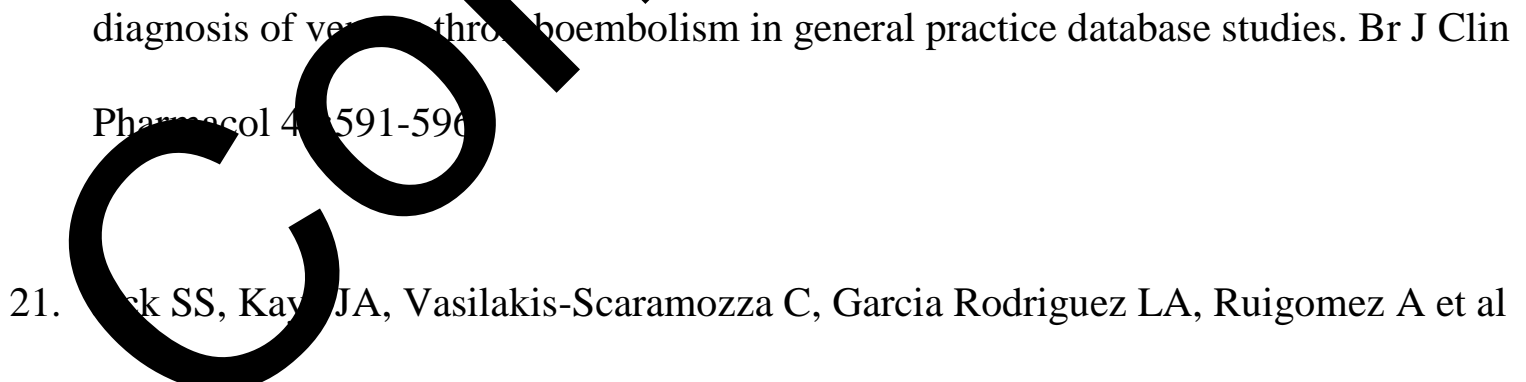
(2003) Validity of the general practice research database. Pharmacotherapy 23:686-689.

22. Opatrny L, Dell'Aniello S, Assouline S, Suissa S (2008) Hormone replacement therapy use and variations in the risk of breast cancer. BJOG 115:169-175. 
23. Gardner DM, Murphy AL, O'Donnell H, Centorrino F, Baldessarini RJ (2010) International consensus study of antipsychotic dosing. Am J Psychiatry 167:686-693.

24. Hankinson SE, Willett WC, Michaud DS, Manson JE, Colditz GA et al (1999) Plasma prolactin levels and subsequent risk of breast cancer in postmenopausal wome Natl Cancer Inst 91:629-634.

25. Tworoger SS, Eliassen AH, Rosner B, Sluss P, Hankinson SE 4) concentrations and risk of postmenopausal breast cancer. G Re 4.6814-6819.

26. Tworoger SS, Sluss P, Hankinson SE (2006) As octą be plasma prolactin concentrations and risk of breast cancer ang a domiv ely premenopausal women.

Cancer Res 66:2476-2482.

27. Tworoger SS, Hankinson SE (2 \&) Pro ctin and breast cancer etiology: an epidemiologic perspective. J Mamp y Glan Biol Neoplasia 13:41-53.

28. Liebrman J Stroup S, McEvoy JP, Swartz MS, Rosenheck RA et al (2005) Effectivenes of antupsychotic drugs in patients with chronic schizophrenia. N Engl J Med 
Female patients from up-to-standard practices prescribed antipsychotic agents

$$
(\mathrm{n}=139,863)
$$

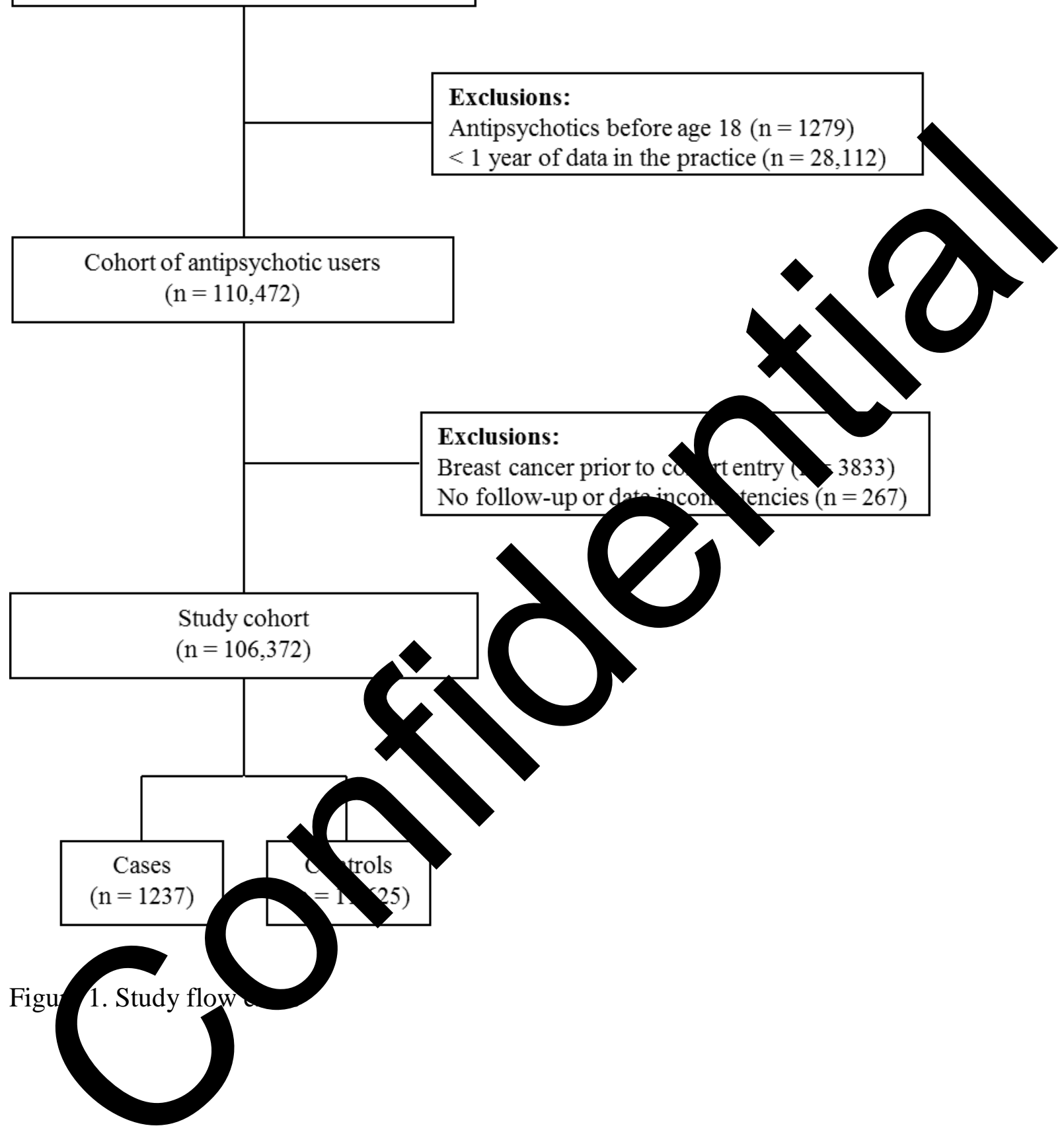




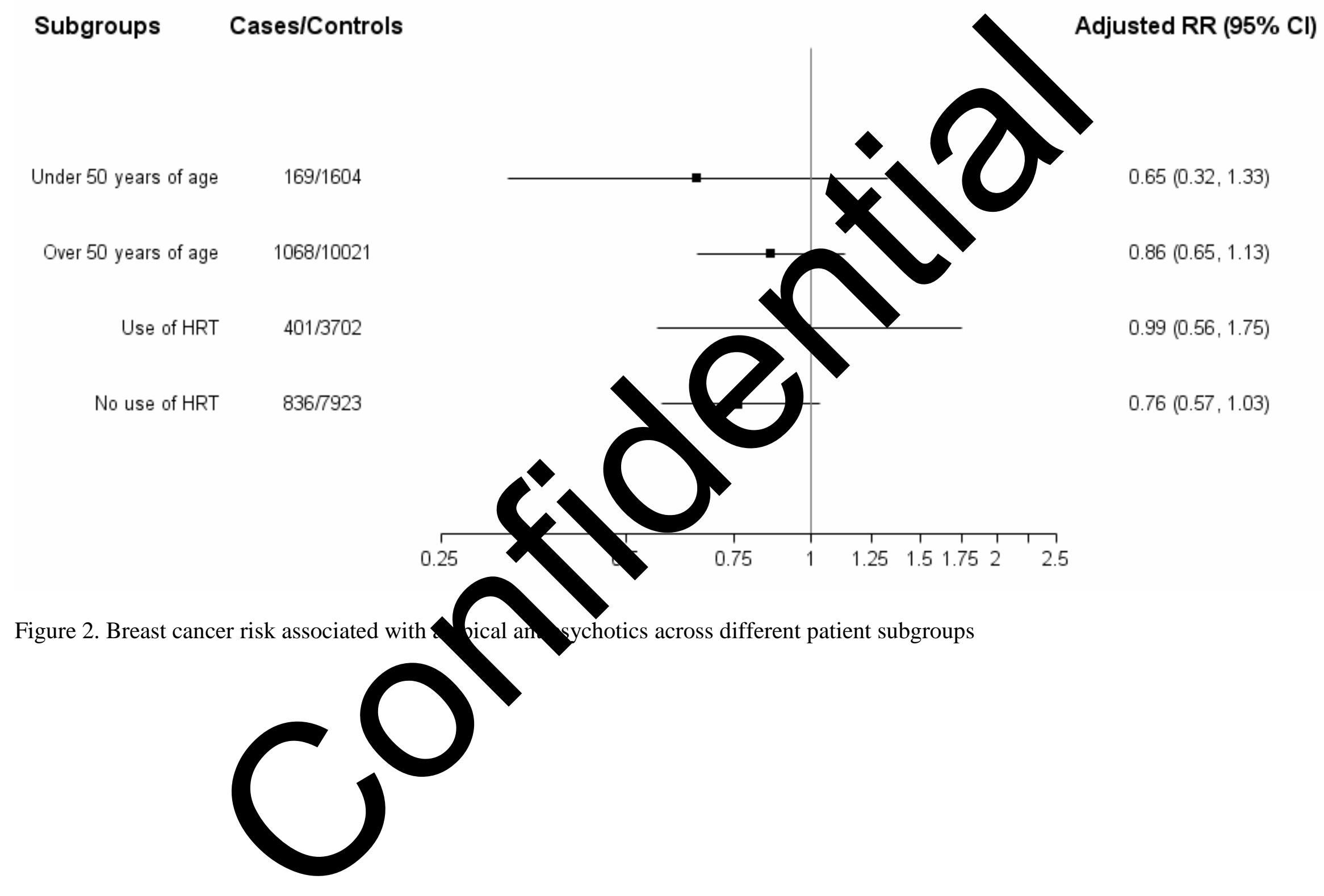


Table 1. Characteristics of cases and controls at index date

\begin{tabular}{lcc}
\hline & $\begin{array}{c}\text { Cases } \\
(\mathbf{n}=\mathbf{1 2 3 7})\end{array}$ & $\begin{array}{c}\text { Controls } \\
(\mathbf{n}=\mathbf{1 1}, \mathbf{6 2 5})\end{array}$ \\
\hline Age (years), mean (SD)* & $66.9(14.3)$ & $66.8(14.1)$ \\
Duration of follow-up (years), mean (SD)* & $7.8(4.8)$ & $7.8(4.7)$ \\
Excessive alcohol use, n (\%) & $105(8.5)$ & $1026(8.8)$ \\
Body mass index, n (\%) & & \\
$\quad<30$ & $751(60.7)$ & $7230(62.2)$ \\
$\quad \geq 30$ & $265(21.4)$ & $2503(21.5)$ \\
$\quad$ Unknown & $221(17.9)$ & $1892(16.3)$
\end{tabular}

Smoking status, n (\%)

Ever
Never

$550(44.5) \quad 5099(43.9)$

Unknown

Aspirin, n (\%)

Selective serotonin reuptake inhibitors, n (\%)

Statins, n (\%)

Previous cancer, n (\%)

Hypertension, n (\%)

Insulin, n (\%)

Metformin, n (\%)

Other oral hypoglycemic agents, n (\%)

Oophorectomy, n (\%)

$557(45.0) \quad 5281(45.4)$

Hormone replacement therar $\mathrm{n}(\%)$

Oral Contraceptives, n (

*Cases and controls matched

$130(10.5)$

$326(26.4)$

$616(49$

$224(18$

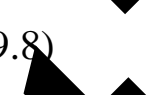

$1245(10$

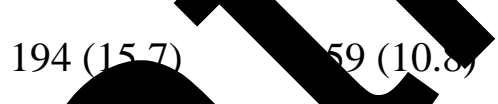

39 (31.9) $336+(29.0)$

2. 8) 94 (1.7)

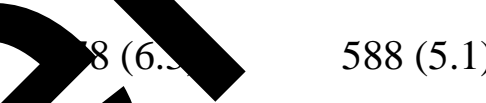

794 (6.8)

354 (3.0)

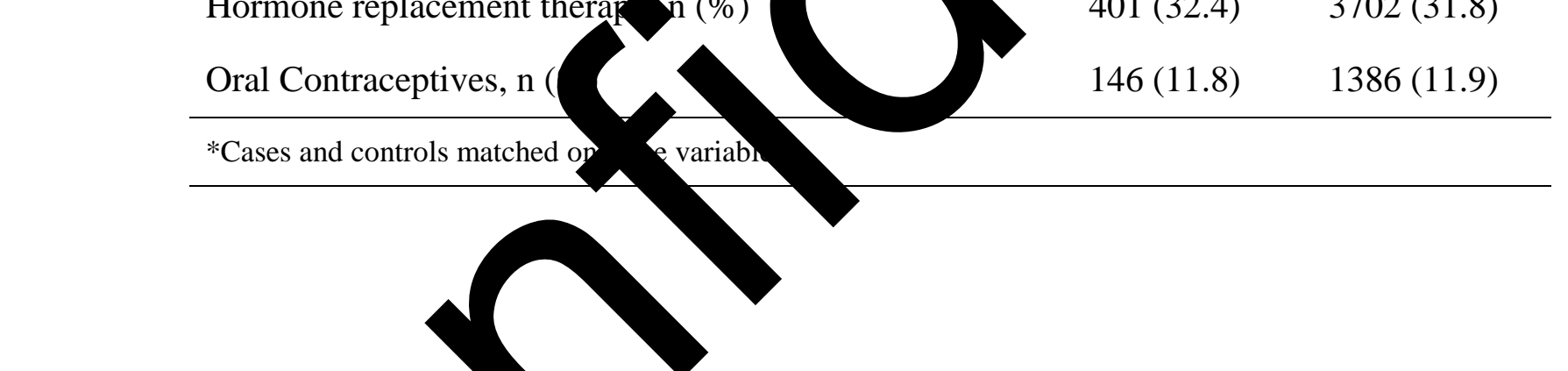


Table 2. Atypical antipsychotics and the risk of breast cancer

\begin{tabular}{lcccc}
\hline & $\begin{array}{c}\text { Cases } \\
(\mathbf{n = 1 2 3 7})\end{array}$ & $\begin{array}{c}\text { Controls } \\
(\mathbf{n = 1 1 , 6 2 5})\end{array}$ & Crude RR & Adjusted RR (95\% CI) \\
\hline Typical antipsychotics only, n (\%) & $976(78.9)$ & $9090(78.2)$ & 1.00 & $1.00($ Reference) \\
Atypical antipsychotics only, n (\%) & $96(7.8)$ & $1078(9.3)$ & 0.82 & $0.81(0.63,1.05)$ \\
$\quad$ Risperidone only, n (\%) & $36(2.9)$ & $386(3.3)$ & 0.87 & $0.86(0.60,1.25)$ \\
$\quad$ Risperidone and other atypical antipsychotics agents, n (\%) & $44(3.6)$ & $479(4.1)$ & 0.83 & $0.81(0.58,1.15)$ \\
$\quad$ Other atypical antipsychotic agents, n (\%) & $16(1.3)$ & $213(1.8)$ & 0.69 & $0.68(0.39,1.19)$ \\
Switches between typical and atypical antipsychotics, $\mathrm{n}(\%)$ & $165(13.3)$ & $1457(12.5)$ & 1.04 & $0.99(0.82,1.20)$ \\
\hline
\end{tabular}

Adjusted for the variables listed in Table 1.

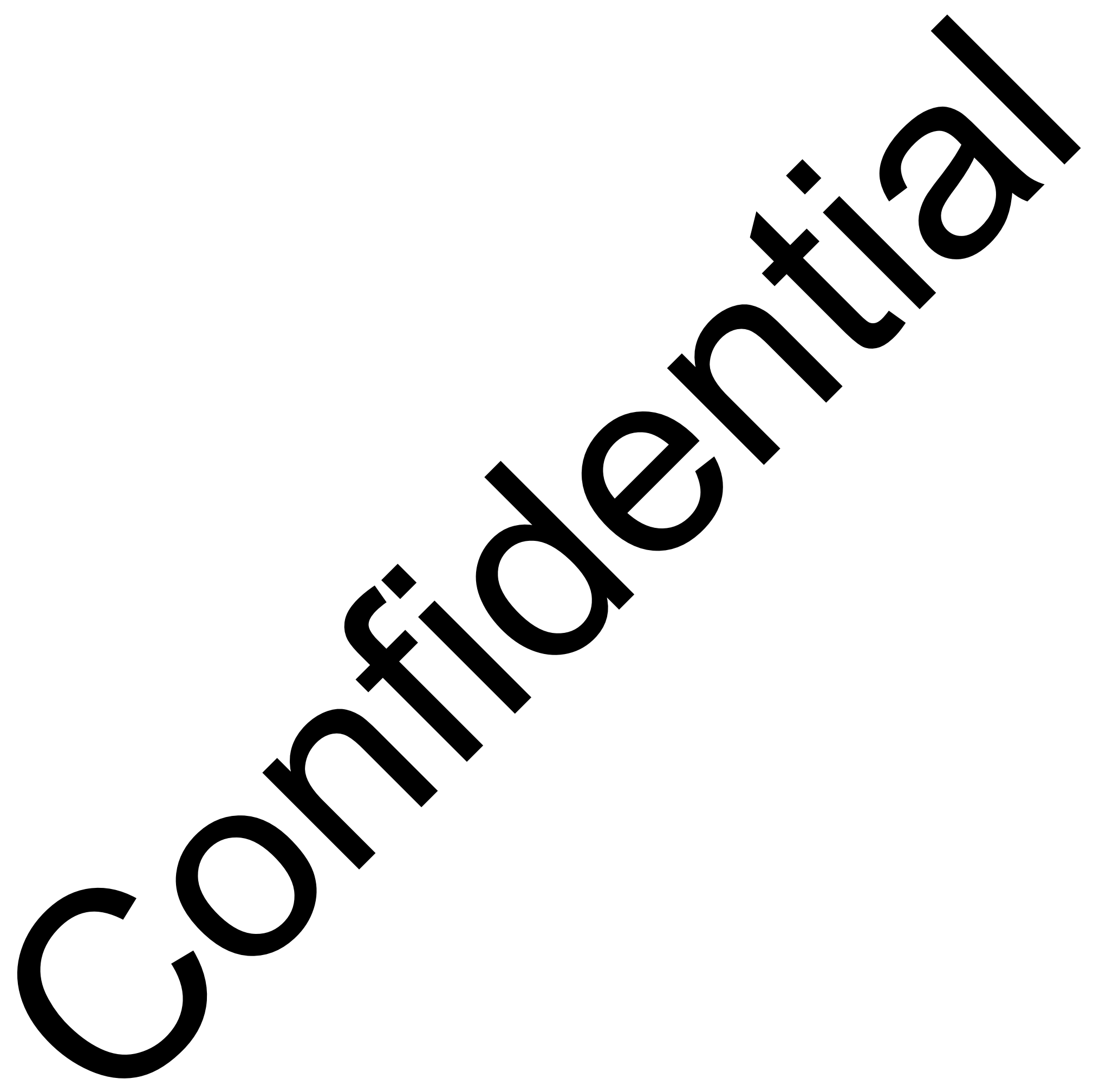


Table 3. Cumulative duration and cumulative dose of atypical antipsychotics and the risk of breast cancer

\begin{tabular}{|c|c|c|c|c|}
\hline & $\begin{array}{c}\text { Cases } \\
(n=1237)\end{array}$ & $\begin{array}{c}\text { Controls } \\
(n=11,625)\end{array}$ & Crude RR & Adjusted RR $(95 \% \text { CI })^{\ddagger}$ \\
\hline Typical antipsychotics only, n (\%) & $976(78.9)$ & $9090(78.2)$ & 1.00 & 1.00 (Reference) \\
\hline \multicolumn{5}{|l|}{ Atypical antipsychotics only } \\
\hline \multicolumn{5}{|l|}{ Cumulative duration of use, $\mathrm{n}(\%)^{*}$} \\
\hline$\leq 224$ days & $36(2.9)$ & $355(3.1)$ & 0.95 & $0.95(0.65,1.39)$ \\
\hline $224-687$ days & $30(2.4)$ & $366(3.1)$ & 0.74 & $0.73(0.48,1.11)$ \\
\hline$\geq 687$ days & $30(2.4)$ & $357(3.1)$ & 0.77 & $0.75(0.50,1.13)$ \\
\hline \multicolumn{5}{|c|}{ Cumulative dose (in olanzapine equivalents), $\mathrm{n}(\%)^{*}$} \\
\hline$\leq 910 \mathrm{mg}$ & $32(2.6)$ & $354(3.0)$ & 0.84 & $0.85(0.57,1.26)$ \\
\hline $910-3965 \mathrm{mg}$ & $31(2.5)$ & $369(3.2)$ & 0.77 & $0.76(0.51,1.13)$ \\
\hline$\geq 3965 \mathrm{mg}$ & $33(2.7)$ & $355(3.1)$ & 0.84 & $(0.56,1.20)$ \\
\hline
\end{tabular}

${ }^{\sharp}$ Adjusted for the variables listed in Table 1.

*Based on tertile categories. 